\title{
Supplementary information: A trans acting ribozyme that phosphorylates exogenous RNA
}

\author{
Sequences \\ 5' GGACCCUA gggaaaagcgaaucauacacaaga ... ... gggcauaagguauuuaauuccaua 3 ' \\ FAMILY 1 (7) \\ 2PT3.1। GTCTTGG*AATAACCCAATAAGGGACGAGACGTGTGAACCAACAACTGACGGATGATTTTTGGGTGCGAACTA \\ 2PT3.19| GTCTTGGCAATAACCCAATAAGGGACGAGACGTGTAAAC-AACTGACGGATGATT-TTTCCGGGTGCGAACTA \\ 2PT3.26| GTCTTGGCAATAACCCAATAAGGGACGAGACGTGTAAAC*AACTGACGGATGATT---TCAGGGTGCG-ACTA \\ 2PT3.4| GTCTTGGCAATAACCCAATAAGGGACGAGGCGTGTAAACCAACTGACGGATGATT---TTTGGGTGCGAACTA \\ 2PT3.29| GTCTTGGCAATAACCCAATAAGGGACGAGACGTGTAA-CAACTGACGGATGATT----TT-GGGTGCGAACTA \\ 2PT3.9| GTCTTGGCAATAACCCAATAAGGGACGAGACGTGTAAACAACTGACGGATGATT----TTTGGGTGCGAACTA \\ 2PT3.27| GTCTTGGCAATAACCCAATAAGGGACGAGACGTGTAA*CAACTGACGGATGATT----TCAGGGAGCGAACTA
}

FAMILY 2 (5)

2PT3.23| GTGCAACGTTATAAGCGTTGACAACAGGACGACGAAAACTGCGAGTGTGATGTGAGTAACGTGCGAACTA

2PT3.15| GTGCAACTGTATAAGCGTTGACAACAGGACGACGAAAACTGCGAGTGTTATGT (partial sequence)

2PT3.12| GTCG-ACGTTATAAGCGTTGACAACAGGACGACGAAAACAGCGAGTGTGATGTGAGTAACGTGCGAACTA

2PT3.13| GTCG-ACGTTATAAGC* TTGACAACAGGACGACGAAAACTGCGAGA* *GATGTGAGTAACGTGCGAACTA

2PT3.21 GTCG-ACGTTATAAGCGTTGACAACAGGACGACGAAAACTGCGAGTGTGATGTGAGTAACGTGCGAACTA

FAMILY $3(4)$

2PT3.20। AGTGTGA*CGTACCTGTGTAAAGATAACGAGAAGCTTCAAACTCTAACTAAGTTTCGGCGCACATCG-CTA 2PT3.25| AGTGTGAACGTACCTGTGTAAAGATAACGAGAAGCATCAAACTCTAACTAAGTTTCGGCGCACATCGACTA 2PT3.30| AGTGTGA*CGTACCTGTGTAAAGATAACGAGAAGCTTCAAACTCTAACTAAGTTTCGGCGCACATCGACTA 2PT3.24| TGTGTGA-CGTTCCTGTGTGATGATAACGAGAAGCTTCAAACTCTAACTAAGTTTCGGCGCACATCGACTA

FAMILY 4 (3)

2PT3.7| CACGACGGAATGCACGTACCAGGACTTTGTCTT-GGGACGTTGGATCATGCCGCGCAA-CGTGCGAACTA

2PT3.10| CACGACGAATGCTTCGTACCAGGACTTTGTCTTTGGGACGTTGGATCATGCCGCGCAATCGTGC-AACTA

2PT3.18| CACGACGAATGCTTCGTACCAGGACTTTGTCTTT-GGACGTTGGATCATGCCGCGCAATCGTGGCAACTA

FAMILY $5(3)$

2PT3.11| CACGATGA-CGTACCACGGAACAAGTGATCACGACTCATGTCGAATGGATGGGTTAACGTGCGAACTA 2PT3.28| CACGATGA-CGTACCAAGGAACAAGTGATCACGACTCATGTTGAATGGATGGGTTAACGTGCGAACTA

2PT3.8| CACGATGA-CGTACCAAGGAACAAGTGATCACGACTCATGAAGAATGGATGGGTTAACGTGCGAACTA

FAMILY $6(1)$

2PT3.5। ACTAGAGTTGAATGAACAAGGTCTGCCTTCATCGATTGTACTTGGGCGATATGGGTAACGTGCGAACTA

FAMILY 7 (1)

2PT3.14| TGCTTCGTACCAGGACTTTGTCTTTGGGACGTTGGAATCATGCCGGAATCGTGGCAACTAGG

Figure 1: Sequences: Selected RNAs from round 12* were reverse transcribed to make cDNAs and cloned into a plasmid vector (pCR2.1 from Invitrogen) for sequencing. The consensus sequences 5, TGCGAACTA 3' (shown in bold) was present in five of the sequence relatedness families. 
A

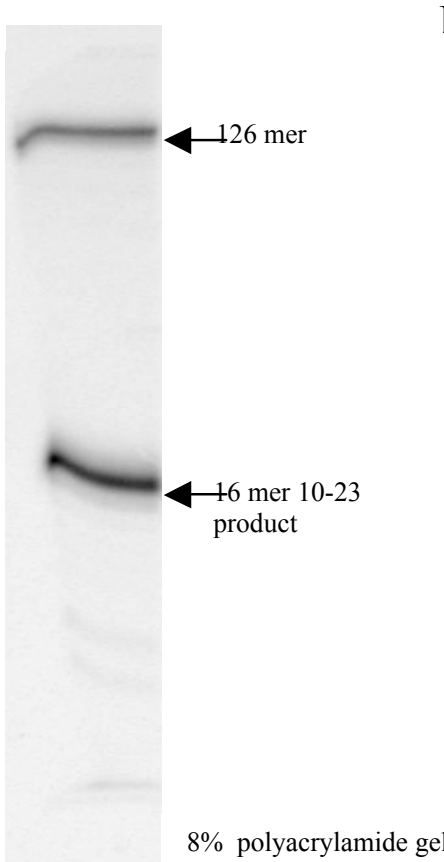

B

Primer
126 mer self $\mathrm{P}-$ RNA
126 mer RNA
118 mer RNA
RT
RNase after RT

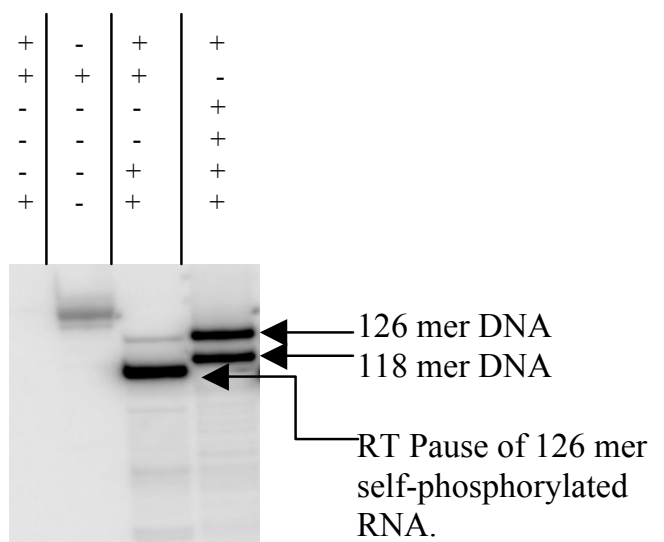

RNA.

Figure 2: Diagnostic assay: A) Cam linked DNA/RNA hybrid molecules were self-phosphorylated using $\gamma^{32} \mathrm{P}$ ATP under conditions similar to those used during the selection. Products were ethanol precipitated, resuspended and annealed with 10-23 DNAzyme. After allowing for DNAzyme-catalyzed cleavage for 6 hours in $20 \mathrm{mM} \mathrm{MgCl}_{2}, 50 \mathrm{mM}$ Tris- $\mathrm{HCl} \mathrm{pH} 7.5$, samples were separated on $12 \%$ polyacrylamide gel, dried and exposed to a phosphorimager screen. Retention of radioactivity on the 16 mer 5 ' cleavage product demonstrates the phosphorylation site to be within that region. B) Internally radiolabeled, autothiophosphorylated transcript $(1 \mu \mathrm{M})$ was recovered from APM gels and annealed to a 5' radiolabeled DNA primer $(10 \mu \mathrm{M})$ that binds to the 3' end of the RNA. Reverse transcription reactions were carried out in 1X MMLV buffer using $200 \mu \mathrm{M}$ dNTPs and Moloney murine leukemia virus reverse transcriptase at $37^{\circ} \mathrm{C}$ for $2 \mathrm{~min}$. The pause product shown above chased into product when reactions carried out for significantly longer times (not shown). Following the reaction, RNA was degraded with RNaseA and reaction mixture was separated on a denaturing $20 \%$ polyacrylamide gel. Two other RNAs (118 and $126 \mathrm{nt}$ ) were included in a separate reaction to control for size of the cDNA product. The single modificationdependent RT pause product is slightly smaller than the $118 \mathrm{nt}$ DNA marker, suggesting that the modification site must lie within the RNA portion between positions +1 and +7 (GGAAAA). 